\title{
La vacunación antigripal reduce consultas, internaciones y mortalidad en personas mayores con enfermedad pulmonar crónica
}

Relation between influenza vaccination and outpatients visits, hospitalization, and mortality in elderly persons with chronic lung disease. Nochol KL, Baken L, Nelson A.Ann Intern Med 1999;130:397-403.

\section{Objetivo}

Definir los efectos de la gripe y los beneficios de la vacunación antigripal en personas mayores con enfermedad pulmonar crónica.

\section{Diseño}

Estudio retrospectivo de cohortes. *

\section{Lugar}

Group Health, sistema gerenciado de salud (250 000 miembros) en Minneapolis, EE.UU.

\section{Pacientes}

Toda persona $\geq 65$ años con diagnóstico previo de enfermedad pulmonar crónica ( $n=1898: 1336$ vacunados y 532 no vacunados).

\section{Medición de resultados principales}

Número de internaciones por neumonía y gripe y también por problemas respiratorios agudos y crónicos; mortalidad global entre personas vacunadas y no vacunadas durante 1993 a 1996. Los datos fueron extraídos de las bases de datos administrativas. Las consultas ambulatorias por dichas causas constituyeron un resultado secundario.

\section{Resultados principales}

Las tasas de vacunación fueron mayores del $70 \%$ y las cepas salvajes y vacunales tuvieron buena concordancia en cada una de las tres estaciones de gripe. Durante éstas, los no vacunados tuvieron el doble de internaciones por neumonía o gripe que entre estaciones ( 111 vs 55 $\%$ personas-año respectivamente, p 0,001 ). Este aumento estacional no existió entre los vacunados ( 45 vs $41 \%$ personas-año, $p=0,11$ ). El Riesgo Relativo (RR) de internación entre los vacunados fue 0,48 (IC95\% 0,28-0,82) y el RR de muerte de 0,30 (IC95\% 0,21-0,43) durante las estaciones de gripe. Estos resultados estuvieron ajustados por edad, sexo, vacunación antineumocóccica, otras enfermedades crónicas, historia de neumonía y número de consultas médicas. La vacunación antigripal también se asoció con menor número de consultas por neumonía, gripe y por todos los problemas respiratorios.

\section{Conclusiones}

En personas mayores con enfermedad pulmonar crónica la gripe se asocia con significativos efectos adversos sobre la salud. La vacunación antigripal los previene y reduce las consultas ambulatorias, las internaciones y la mortalidad.

Fuente de financiamiento: Group Health Foundation.

\section{COMENTARIO}

La gripe continúa siendo una importante causa de morbimortalidad y las personas mayores con enfermedad pulmonar crónica constituyen un grupo particularmente de alto riesgo. La tasa de internación de neumonía es 2-7 veces mayor que en las personas sin esa condición. ${ }^{1-2}$

Más del $90 \%$ de las muertes por neumonía y gripe durante epidemias se dan entre personas $\geq 65$ años $y$ a pesar de que hace tiempo que existe recomendación de vacunación antigripal a este grupo, por su demostrada costo-efectividad ${ }^{3}$, un $35 \%$ de éstos no está vacunado en EEUU 4 y la cifra es mucho mayor en los países en desarrollo. Un $12 \%$ de los $\geq$ 65 años padece enfermedad pulmonar crónica y en ellos la cobertura antigripal también es inadecuada. Este estudio confirma, a través de una gran cohorte y en estaciones consecutivas, la efectividad de la vacunación antigripal en un grupo específico de alto riesgo, reduciendo $52 \%$ las internaciones relacionadas y $70 \%$ la mortalidad global, en consistencia con otros pequeños estudios de cohortes. ${ }^{5}$ El estudio tuvo un gran poder estadístico para detectar diferencias no solo por el alto $" \mathrm{~N}^{\prime \prime}$ sino también por el gran número de eventos (cuatro veces más internaciones que los pacientes sin enfermedad pulmonar crónica), pero tiene las timitaciones propias de los estudios observacionales.* Aunque tuvo un adecuado ajuste por otras variables de salud, quizás two haya sido suficiente, dadas las marcadas diferencias basales entre wacunados y no vacunados y por no haber incluido información sobre estilo de vida (especialmente tabaquismo), estado funcional y socioecanómico, que podrían haber influido tanto en la decisión de vacunar como en los resultados. Sin embargo ni las internaciones por neumonía entre estaciones, ni las internaciones por causas no-cardiopulmonares difirieron significativamente entre los grupos, sugiriendo, al menos, la ausencia de desbalances insalvables. Los errores de registro en las bases de datos podrían timitar este estudio, pero esto es poco probable dado que el grado de acuerdo reportado con las historias clínicas y otras bases de datos es del $93 \%$. Lo que sí debería tomarse con cautela es el RR de internaciones por neumonía y gripe, ya que a pesar del importante tamaño muestral existen amplios intervalos de confianza (menor precisión en la estimación). Diferentes estudios controlados mostraron $70-80 \%$ de efectividad en prevenir la gripe o reducir su severidad y solo parece disminuir en los $\geq$ de 70 años. De cara a la actual estación de gripe es importante recordar las indicaciones de vacunación además de los $\geq 65$ años: pacientes con enfermedades crónicas cardiorespiratorias (niños > 6 meses), con enfermedades metabólicas (incluida diabetes), hemoglobinopatías, inmunosupresión (incluido VIH), insuficiencia renal y personal de salud o convivientes en contacto con este grupo de pacientes. Aunque con resultados menos impactantes, también podría considerarse en internados en $3^{\text {er nivel, emba- }}$ razadas en $2^{\text {do }}$ y $3^{\text {er }}$ trimestre durante época de epidemia y en personas jóvenes que deseen vacunarse (en estos últimos disminuyen un $25 \%$ la infecciones respiratorias altas y un $43 \%$ el ausentismo laboral por estas causas). ${ }^{6}$

Dr. Agustín Ciapponi

Unidad de Medicina Familiar y Preventiva. Hospital Italiano de Buenos Aires.

Fefferencias

- Fister DA, Talsma A, Furumoto-Dawson A et al. Influenza vaccine effectiveness in preventing hospitalization among the elderty. Am J Epidemil. 1992;136:296-307.

2 Ihrmit 5e, Monto AS. Influenza vaccine effectiveness in preventing hospitalization among the elderly during influenza type A and type B seasons. Int J Epidemiol. 1995;24:1240-8.

- Vifichol, KL, Margolis KL, Wuorenma J, et al. The efficacy and cost effectiveness of vaccination against influenza among elderly persons living in the community. $N$ Engl $J$ Med IIS. $331: 778-84$.

- Thesumococcal and influenza vaccination levels among adults aged $\geq 65$ years -United States, 1997 MMWR Morb Mortal Wkly Rep 1998;47:797-802

A Aawi. wS, Cowan J Jackson GG. Acute respiratory illness among inmunized and noninmunized patients with high risk factors during a split season of influenza A and B. J Inf Dis. wate $157.633-9$

Ai vichal. KL, Lind A, Margolis KL, et al. The effectiveness of vaccination against influenza in healthy, working adults. N Engl J Med 1995;333:889-93. 